\title{
Welcome to the year 2017
}

\author{
Nadia Magnenat-Thalmann ${ }^{1}$
}

Published online: 10 January 2017

(C) Springer-Verlag Berlin Heidelberg 2016

The Visual Computer Journal is progressing well. We have increased our Impact Factor, and we have received more than 550 submissions during the year. Our acceptance rate is about 23\%. Each submitted paper is handled by an Associate Editor (AE) that finds at least 3 reviewers to review the paper. I take the opportunity to thank all of them for their tremendous and invaluable work.

Throughout 2016, some Associate Editors have left the editorial board of the Visual Computer. I would like to thank them for their great work and contribution.

Here is the list of the Associate Editors who have left the editorial board:

- Vaclav Skala, University of West Bohemia, Plzen, Czech Republic

- Scott Schaefer, Texas A\&M University, USA

- Eftychios Sifakis, University of Wisconsin-Madison, USA

- Dimitri Van De Ville, University of Geneva/Ecole Polytechnique Fédérale de Lausanne, Switzerland

- Gerald Farin, Arizona State University USA

- Sung-Eui Yoon, KAIST, Korea

- Hongbo Fu, City University of Hongkong, China

- Mark Grundland, Functional Elegance, UK.

A special thought goes to Gerald Farin. Gerald Farin passed away in January 2016. For the Visual Computer and of course for all colleagues and students, it is a real loss. Each time we had a complex paper to review in geometric modeling, I have sent it to him. I was then sure that the paper

Nadia Magnenat-Thalmann

thalmann@miralab.ch

1 MIRALab-CUI, University of Geneva, Battelle, Building A, 7, Route de Drize, 1227 Carouge, Geneva, Switzerland will be deeply reviewed and that I always could rely on his judgment. He has served the Visual Computer Journal almost since its beginning. We will miss him a lot, and I will always be grateful to him for his tremendous contribution.

I would like to welcome the new Associate Editors who have joined the editorial board during 2016:

- Ligang Liu, University of Science and Technology of China, China

- Amir Vaxman, Utrecht University, The Netherlands

- Yaron Lipman, Weizmann Institute of Science, Israel

- Marc Christie, University of Rennes 1, France

- Mathias Zwicker, University of Bern, Switzerland

- Bruno Levy, Inria, France

- Richard Zhang, Simon Fraser University, Canada

- Adrien Bousseau, Inria Sophia-Antipolis, France

- Nobuyuki Umetani, Autodesk Research, Canada

- Matthew Kyan, York University, Canada

- Bochang Moon, Gwangju Institute of Science and Technology, Korea

- Rémi Cozot, IRISA, University of Rennes 1, France

- Li Liu, National University of Defense Technology, China

- Zerrin Yumak, University of Utrecht, Netherlands

- Youyi Zheng, Shanghai Tech, China

- Nicolas Bonneel, Universite of Lyon, France.

The editorial board of the Visual Computer is renewed regularly, and we are happy that these new AEs in various fields will handle 3 to 5 papers a year in their field.

This year, we have introduced new topics and new keywords for the Visual Computer: We expect more papers on 3D fabrication, social robotics, affective computing, and deep learning algorithms. We invite the research community to propose special issues in these topics. Our new list of key- 
words allows a better dispatch of the papers. The author can then choose more precise keywords as well as the Associate Editors. We hope to improve the adequate selection of the reviewers for each paper.

During 2017, the journal will publish the 35 best papers of the Computer Graphics Conference (CGI'2017) that will be held in Japan in June 2017. We will also publish the very few best papers of other conferences as Cyberworlds, VS-Games, and 3DOR.

I would like to thank the Editorial Assistants: Aryel Beck and Jan Perhac, for their collaboration in the management of the journal over the year 2016 and welcome to our new Editorial Assistant Juzheng Zhang.
Since last year, the journal is managed with the collaboration of three Associate-Editors-in-Chief: Daniele Panozzo, from New York University in USA, Hyewon Seo, from CNRS France and Hui Huang, from Shenzhen University in China. I thank them for their tremendous support and collaboration. With different know-how on board, the papers can be handled more adequately.

Finally, this journal could not exist without the strong support of our Editorial Director at Springer, Beverley Ford, as well as Rachel Roberts, the Editor of the journal. Thank you also to Beate Uhl, the Production Editor, and also to Sneha Rahul for their continuous work and assistance. 